\title{
Corela
}

Cognition, représentation, langage

13-2 | 2015

Vol. $13, n^{\circ} 2$

\section{Le koulango, une langue gur à deux genres}

\section{Zakari TCHAGBALÉ et Kouakou Appoh Enoc KRA}

\section{OpenEdition}

\section{Journals}

Édition électronique

URL : http://journals.openedition.org/corela/4141

DOI : $10.4000 /$ corela.4141

ISSN : 1638-573X

\section{Éditeur}

Cercle linguistique du Centre et de l'Ouest - CerLICO

\section{Référence électronique}

Zakari TCHAGBALÉ et Kouakou Appoh Enoc KRA, «Le koulango, une langue gur à deux genres », Corela [En ligne], 13-2 | 2015, mis en ligne le 01 décembre 2015, consulté le 30 avril 2019. URL : http:// journals.openedition.org/corela/4141; DOI : 10.4000/corela.4141

Ce document a été généré automatiquement le 30 avril 2019

\section{(c) (i) (2)(2)}

Corela - cognition, représentation, langage est mis à disposition selon les termes de la licence Creative Commons Attribution - Pas d'Utilisation Commerciale - Partage dans les Mêmes Conditions 4.0 International. 


\title{
Le koulango, une langue gur à deux genres
}

\author{
Zakari TCHAGBALÉ et Kouakou Appoh Enoc KRA
}

\section{Introduction}

1 Depuis William H. J. Bleek (1827-1875) certaines langues africaines comme le koulango sont qualifiées de «langue à classes». La classe nominale regroupe autour d'un affixe plusieurs radicaux nominaux. Une langue à classes en compte plusieurs, certains pour la forme singulière du nom, d'autres pour la forme plurielle. Le couple d'affixes singulier/ pluriel constitue un genre. L'affixe n'est pas censé renvoyer à une valeur sémantique; le couple d'affixes ne peut donc pas être un genre au sens classique, il est dit grammatical. Cette théorie devenue un classique dans la linguistique africaine n'est pas opératoire : elle exclut l'individu parlant en faisant croire qu'une langue peut générer de manière arbitraire des regroupements de noms; elle s'éloigne de ce qui parait logique en postulant, pour une même langue, plusieurs marqueurs de pluriel; elle demeure dans l'absence de logique en postulant, un marqueur de singulier, qui plus est, plusieurs marqueurs de singulier alors que d'autres langues n'en ont même pas.

2 A la théorie de "la classe nominale ", nous opposons une théorie du genre. Le monde extralinguistique est un véritable chaos. Les objets autour de nous sont disposés sans aucun ordre. D'une certaine façon, l'homme crée des « dossiers " pour les regrouper, par affinité sémantique: dossier des mammifères, dossier des végétaux, etc. Chaque communauté crée ses dossiers en se fondant sur sa connaissance de la nature et sa culture. Les dossiers ne peuvent donc pas être les mêmes d'une communauté à l'autre.

3 Membre d'une communauté linguistique, l'individu découvre ces dossiers et leurs spécificités lors de l'assimilation du langage et de la culture. Certaines langues facilitent le travail à l'apprenant en affectant à chaque dossier un signe censé représenter la propriété sémantique commune aux objets qui y sont rangés. Ce signe que chaque nom est tenu d'afficher est l'affixe nominal. Adossé à une valeur sémantique, il représente à lui 
seul un genre (soit $\mathrm{G}$ ). Comme les dossiers, les genres ne sont pas les mêmes d'une famille linguistique à une autre. Aussi n'est-il pas étonnant que pendant que les langues indoeuropéennes privilégient le critère de sexe (masculin/féminin) dans la création des genres, les langues Niger-Congo privilégient, quant à elles, celui de l'animation (animé/ non-animé) ou de l'humanité (humain/non-humain). Le marqueur de genre $G$ peut être préfixé comme en bantu et en kwa ou suffixé comme en ouest-atlantique et en gur.

En présence de $G$, la forme désignative devient un radical (Rad) qui sélectionne un affixe pour être un nom. La forme actualisée après affixation, [G-Rad] ou [Rad-G], est ce qui renvoie à la notion de l'objet. Rad en est la partie lexicale et $G$ la partie grammaticale. Rad en donne le sens lexical et $\mathrm{G}$ le classement en genre. C'est à ce nom, l'ensemble [Rad-G], qu'en cas de besoin, s'adjoint le marqueur de pluriel (Pl), en tant que dérivatif.

Tout comme dans une langue sans genres, le dérivatif Pl transforme une unité lexicale qui fonctionnait déjà. En présence de l'affixe de genre, son adjonction peut se faire par concaténation ou par substitution.

Les affixes $\mathrm{G}$ et $\mathrm{Pl}$ des langues gur ont trois schèmes : CV, C et V (Tchagbalé, 2014). Les affixes de schèmes $\mathrm{CV}$ que sont $/ \mathrm{ba} /$ et $/ \mathrm{ka} /$ peuvent, dans certaines langues, perdre leur $\mathrm{V}([\mathrm{a}])$, mais ils auront un traitement qui les distinguera des $\mathrm{C}$ qui ont la même consonne qu'eux. Quant aux affixes $\mathrm{C}$, les langues qui transforment tout schème $\mathrm{CVC}$ en $\mathrm{CVCV}$, leur affectent une voyelle de soutien qui est soit une voyelle choisie au sein du système vocalique de la langue soit une copie d'une voyelle voisine. En koulango, le suffixe C a pour voyelle de soutien une voyelle spécifique, [0] ou une copie de la voyelle du radical.

7 La limitation aux schèmes $\mathrm{CV}, \mathrm{C}$ ou $\mathrm{V}$ concerne aussi bien les affixes $\mathrm{G}$ que les formes de $\mathrm{Pl}$. A part quelques langues, rien ne distingue un affixe $\mathrm{G}$ d'un affixe $\mathrm{Pl}$ en dehors de la fonction assumée.

Le koulango compte quatre affixes de genre. On peut supposer qu'il possède quatre genres distincts comme la plupart des langues gur. Or on n'en observe fonctionnellement que deux. Comment se justifie, d'un point de vue morphosyntaxique, le passage de quatre genres à deux ? Quelle est la valeur sémantique de chacun des deux genres? Qu'est-ce qui justifie la réduction des genres?

9 Pour répondre à ces questions, l'article s'articule en trois parties : la première s'attachera à la mise au jour des affixes des noms koulango et, parmi eux, les affixes de genre; la seconde démontrera le processus de réduction des genres qui aboutit à deux ; la troisième tentera de trouver les raisons sociolinguistiques de la réduction.

\section{Les suffixes de genre du koulango}

\subsection{Technique de mise à jour du suffixe}

Dans une langue à genres, le premier indice de la présence de genres est la différenciation des affixes nominaux. Les noms koulango manifestent des suffixes différenciés. Quels sont-ils? Sont-ils révélateurs de genres? Pour mettre au jour ces affixes, il est bon d'exposer brièvement notre méthodologie. Pour ce faire, il faut rappeler le mode d'adjonction du marqueur du pluriel dans un nom.

11 Dans une langue sans genres, c'est au nom qui fonctionne déjà comme unité morphosyntaxique que s'adjoint le marqueur de pluriel quand l'objet désigné se présente en plusieurs unités. Ainsi, en gen-mina par exemple, le nom jonu« femme » fonctionne 
comme sujet dans la phrase nonuva « Une femme est venue ». C'est comme tel que ce nom reçoit son marqueur de pluriel qui est wo pour devenir nonuwo «femmes ».

Dans une langue à genres, le nom tel qu'il fonctionne en dehors du pluriel est complexe. Il est composé d'un radical et d'un marqueur de genre. En espagnol par exemple, les noms muchacho "garçon» et muchacha «fille» sont composés du radical muchach« enfant » et du marqueur du genre masculin -o pour le premier et du marqueur du genre féminin -a pour le second. C'est avec cette forme complexe qu'ils reçoivent le marqueur de pluriel -s : muchachos « garçons » et muchachas « filles ».

Mais il peut se trouver qu'au lieu de se concaténer au marqueur de genre, le marqueur de pluriel se substitue à lui. Dans ce cas, il prend une forme spécifique pour chaque genre de manière à ne pas en oblitérer l'identité. Observons les suffixes d'accord de l'adjectif de radical bon- "bon" du latin. Avec le genre masculin dont le marqueur est -us, le marqueur de pluriel se présente sous la forme de -i. Ainsi, on a : bon-us «bon » et bon-i «bons ». Avec le genre féminin dont le marqueur est -a, le marqueur de pluriel est -ææ .Ainsi, on a : bon-a « bonne » et bon-æ « bonnes ».

14 Les langues niger-congo à genres font leur pluriel comme le latin. Le marqueur du pluriel se substitue au marqueur du genre et prend une forme spécifique pour chaque genre. Langue gur, le koulango fait comme les autres langues niger-congo. Son marqueur de pluriel se substitue au marqueur de genre avec, à chaque fois une forme spécifique. Ainsi, lorsque le suffixe de genre n'est pas visible à l'œil nu, c'est par la commutation avec le marqueur de pluriel que l'on parvient à le mettre au jour.

Il convient de rappeler que le suffixe koulango est de schème $\mathrm{C}$ ou V. Quand il est $\mathrm{C}$, la langue lui fournit une voyelle de soutien dans le discours. Cette voyelle est dans certains cas une copie de la voyelle du radical et dans d'autres, une voyelle choisie dans le système vocalique pour ces propriétés particulières. Cette dernière est, pour rappel,-o et la même pour tout suffixe.

\subsection{Identification des suffixes}

\section{Le suffixe /l/}

16 Le suffixe /1/ s'affiche sous différentes formes : [1, $\mathbf{r}$ et $\mathbf{n}]$. On trouve la variante [1] dans le

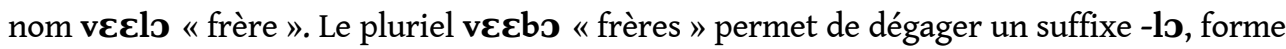
soutenue de /1/.

Quant à la variante $[\mathbf{r}]$, on l'observe dans $\mathbf{j} \boldsymbol{\varepsilon} \mathbf{r} \boldsymbol{\varepsilon}$ 《femme » et mãrã «chien ». Elle est confirmée comme suffixe de genre par la commutation avec les pluriels jebo «femmes » et mãbo « chiens ». Dans les deux noms jerr « femme » et mãrã « chien », la voyelle de soutien est identique à celle du radical. Elle en est donc une copie. La consonne de base /1 / se trouve ainsi pris en sandwich par un même timbre vocalique. Elle se trouve dans une situation d'affaiblissement. Cela peut expliquer pourquoi elle est devenue [r].

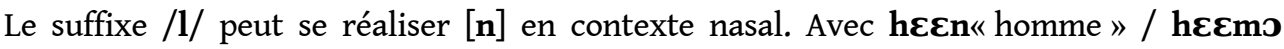

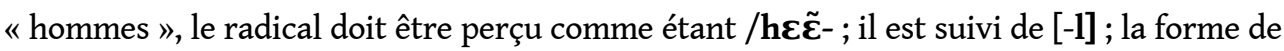

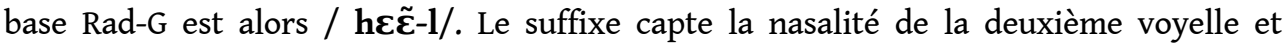

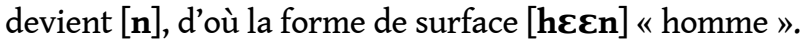




\section{Le suffixe / $\mathrm{k} /$}

19 Les exemples des noms qui suivent : kakכ " arachide ", toko "canne ", d $\boldsymbol{\varepsilon} \boldsymbol{\varepsilon} \mathbf{k J}$ " arbre " présentent explicitement un suffixe / $\mathbf{k}$ / qui commute avec une forme au pluriel, [n], ainsi que le montre la commutation avec les formes plurielle de ces noms : kako/kan, toko/

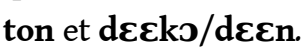

\section{Remarques}

20 1) Il est utile de rappeler que le koulango est une langue sans consonnes nasales phonologiques. La forme [n] du pluriel destinée au suffixe / $\mathbf{k} /$ est donc la variante d'une consonne orale. Dans l'état actuel de la langue, rien ne permet d'identifier cette consonne. Dans d'autres langues gur comme le tem, le même [n] du pluriel est une forme affaiblie de la consonne / $\mathbf{t}$ / qui représente dans cette langue une forme spécifique du pluriel (Tchagbalé, 2010). S'agit-il donc de la variante d'une ancienne forme du pluriel, / t/, aujourd'hui disparue?

21 2) Il est à noter que certains noms d'animaux de suffixes /1/ font leur pluriel, non pas en / b/ mais en [n]. En voici quelques exemples :

\begin{tabular}{|c|c|c|}
\hline puvro & puvn & «poisson $(\mathrm{s}) »$ \\
\hline 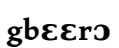 & 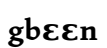 & 《grenouille (s)» \\
\hline buuro & buun & « gazelle (s)» \\
\hline
\end{tabular}

22 Cette anomalie apparente pourrait trouver son explication soit dans un cadre socioculturel, la société considèrerant certains êtres comme dotés d'une propriété qu'ils n'ont pas visiblement, soit dans le cadre d'une évolution d'un système où des noms orphelins sont affectés dans des genres qui n'étaient pas les leur.

\section{Le suffixe /g/}

23 Le suffixe $/ \mathbf{g} /$ a quatre variantes : $[\mathbf{g}, \mathbf{X}, \mathbf{y}$ et $\mathbf{J}]$ imposées par un contexte soit phonique soit morphologique.

Les noms gbigo « jour» et gbokugo «tabouret » présentent la variante /g/, laquelle est confirmée par la commutation avec les pluriels respectifs gbiũ «jours» gbokuũ « tabourets » dotés du marqueur spécifique $-\tilde{\mathbf{v}}$.

Lorsque la voyelle de soutien est une copie de la voyelle du radical, /g/ peut perdre son occlusion et devenir [x]. C'est le cas avec teye « chèvre » ou toðo « feuille de papier ». La fricative peut même disparaître et créer une structure CVV. En témoignent fee « uuf »;

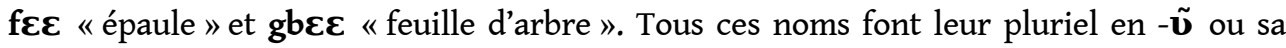

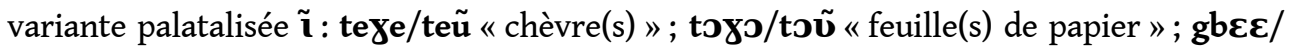

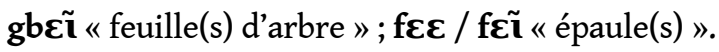

Précédée par une voyelle nasalisée, /g/ se nasalise et devient [y]. C'est le cas dans sãyã «nez » dont le radical est /sã-/ et le plurielsãõ. 
Quand le suffixe /g/ vient sur un radical déjà « élargi » par le dérivatif [1], il devient [\}]. C'est le cas dans parajo « étoile » dont le pluriel parã̃ « étoiles » montre bien que [j] est une variante de $/ \mathbf{g} /$. En contexte nasal, [j] se transforme en [n]. En témoignent fino « lune » réalisation en surface de /fi-l- $\mathbf{- j} /$.

\section{Remarque}

La forme / $\tilde{\mathbf{v}} /$ du pluriel, sert également de marqueur à des noms de suffixe /1/. Le nom [Rad-1] est perçu comme un radical inanalysable ; ce qui explique le maintien du suffixe / 1/ alors qu'il ne joue plus son rôle d'aiguilleur de genre. Exemples :

\begin{tabular}{|c|c|c|}
\hline tulo & tuloũ & " éléphant(s)» \\
\hline zulo & zulov & «pintade(s)» \\
\hline pulo & puloũ & « vautour(s)» \\
\hline
\end{tabular}

\section{Le suffixe /kp/}

La liste des noms suivants propose le suffixe / $\mathbf{k p} /$ :

\begin{tabular}{|l|l|}
\hline kaakpo & « haricot » \\
\hline suukpo & « cure-dent » \\
\hline fekpo & « piège » \\
\hline
\end{tabular}

La commutation avec le pluriel dont le marqueur est [m] confirme l'identité de ce suffixe.

\begin{tabular}{|l|l|l|}
\hline kaakpo & kaam & « haricot(s)» \\
\hline suukpo & suum & « cure-dent(s) » \\
\hline f $\varepsilon$ kpo & f $\varepsilon m$ & « piège $(s) »$ \\
\hline
\end{tabular}

\section{Remarque}

31 L'on sait que toute consonne nasale koulango est une réalisation en surface d'une orale. Ici comme pour le [n], forme de pluriel correspond à $/ \mathbf{k} /$, la nasalité n'est pas due à un contexte nasal mais au probable affaiblissement d'une consonne orale. La réalisation [m] ne pourrait être, en toute hypothèse, que l'affaiblissement de la consonne $/ \mathbf{b} /$. Or $/ \mathbf{b} /$ est la forme du pluriel pour le suffixe /1/. Il faut alors supposer qu'il existe deux formes du pluriel ayant pour consonne /b/. Le /b/ de /l/ devrait correspondre au /ba/ des nombreuses langues gur dans lesquelles on trouve un suffixe /b/ qui se réalise [m] en position suffixale. C'est le cas en sénoufo (Tchagbalé, 2013) et en tem (Tchagbalé, 2010). Il 
est donc envisageable, qu'en koulango,il y ait deux suffixes /b/, l'un d'origine CV, l'autre d'origine $\mathrm{C}$. Ainsi, notre forme de pluriel $[\mathbf{m}]$ serait bien la réalisation de $/ \mathbf{b} /$.

En conclusion, à travers ses quatre suffixes $/ \mathbf{l} /, / \mathrm{k} /, / \mathrm{g} /$ et $/ \mathrm{kp} /$, le koulango donne des indices de la présence de quatre genres. Il reste à les confirmer par l'examen de leur prise en charge dans le discours.

\section{Les genres}

Les noms du koulango se regroupent donc autour de quatre marqueurs de genre, /l/, /k/, $/ \mathrm{g} /, / \mathrm{kp} /$. Théoriquement la langue devrait compter quatre genres. Mais le genre n'existe pas seulement grâce à la présence d'un affixe nominal. Celui-ci n'est qu'un indice et le genre doit être confirmé par l'énonciation dans le discours.

Le regroupement des noms d'une langue par thème sémantique, l'affectation à chaque groupe d'un affixe représentant la valeur sémantique à la base du regroupement, est le travail du constructeur de la langue. Au locuteur, le nom est donné dans sa globalité, radical et affixe combinés. Le travail du locuteur est de mémoriser la forme globale du nom. Dans le discours, le nom est repris dans ses déterminants dépendants tels que ceux de la qualification, de la quantification, de la monstration, de la spécification, etc. Il est repris également par son substitut par excellence, le pronom de $3^{\text {ème }}$ personne. Ici, l'apprentissage des formes de reprise du nom se limite à quelques modèles. Pour le reste, l'énonciateur demeure le maître du jeu: il lit la valeur sémantique du genre moins à travers l'affixe qu'à travers le radical. C'est ce qui lui permet de classer dans le genre approprié un nom d'emprunt ou un nom propre (patronyme, toponyme), dépourvus d'affixe. C'est donc dans le discours que s'actualise le genre.

Les deux instances du discours qui attestent le genre sont donc le déterminant dépendant (Dt) et le pronom. C'est sur ces deux instances que nous allons tester les genres que supposent les quatre suffixes du koulango. Voici un échantillon où chaque nom porte un des suffixes.

\begin{tabular}{|c|c|c|c|}
\hline & & $\mathrm{Dt}$ & \\
\hline /1/ & 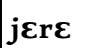 & biiro & «femme noire» \\
\hline /k/ & kako & biiko & « arachide noire » \\
\hline /g/ & toyo & biiko & « feuille de papier noire» \\
\hline$/ \mathrm{kp} /$ & fekpo & biiko & «piège noir » \\
\hline
\end{tabular}

Sans la ligne /1/ où le suffixe du déterminant es [ro] et non [ko], on aurait pu poser qu'il n'y a pas d'accord, que l'adjectif "noir» a une forme fixe unique, biiko. On peut également considérer qu'il y a bien un accord mais qu'il est limité à deux affixes, /1/ et / $\mathbf{k}$ $/$, les affixes $/ \mathbf{g} /$ et $/ \mathbf{k p} /$ s'alignant sur $/ \mathbf{k} /$.

Quand on remplace le déterminant à radical /bii/ «noir » par un déterminant non lexical, le défini, on observe le même phénomène : 


\begin{tabular}{|l|l|l|l|}
\hline & & Dt & \\
\hline$/ \mathbf{l} /$ & $\mathbf{j} \boldsymbol{\varepsilon} \mathbf{r} \boldsymbol{\varepsilon}$ & $\mathbf{n}$ & « la femme en question » \\
\hline$/ \mathbf{k} /$ & $\mathbf{k a k o}$ & $\mathbf{r} \boldsymbol{\varepsilon}$ & « l'arachide en question » \\
\hline$/ \mathbf{g} /$ & $\mathbf{t o y}$ & $\mathbf{r} \boldsymbol{\varepsilon}$ & « la feuille de papier en question » \\
\hline$/ \mathbf{k p} /$ & $\mathbf{f} \boldsymbol{\varepsilon} \mathbf{k p o}$ & $\mathbf{r} \boldsymbol{\varepsilon}$ & « le piège en question » \\
\hline
\end{tabular}

A l'affixe /l/ correspond la forme [n] et aux affixes $/ \mathrm{k} /, / \mathrm{g} /, / \mathrm{kp} /$ correspond la forme [ $\mathbf{r \varepsilon}$.

Au niveau du déterminant (adjectif qualificatif, marqueur du défini), il y a bien accord, mais un accord réduit. La seconde instance du discours qui pourrait manifester un accord avec le nom est le pronom. Examinons sa ou ses formes après chacun des quatre suffixes :

\begin{tabular}{|l|l|l|l|l|l|}
\hline & & & Pronom & & \\
\hline$/ \mathbf{l} /$ & $\mathbf{j} \varepsilon \mathbf{r} \varepsilon$ & $:$ & $\mathbf{h} \tilde{\mathbf{v}}$ & cei & « elle est tombée » \\
\hline$/ \mathbf{k} /$ & kako & $:$ & $\mathbf{h o}$ & cei & « elle est tombée » \\
\hline$/ \mathbf{g} /$ & toxo & $:$ & $\mathbf{h o}$ & cei & « elle est tombée » \\
\hline$/ \mathbf{k p} /$ & fekpo & $:$ & ho & cei & « il est tombé » \\
\hline
\end{tabular}

On a affaire à un phénomène déjà relevé avec le déterminant. Le pronom a la forme [ho] partout sauf avec l'affixe /1/ où il est réalisé [hũ].

$41 \mathrm{Au}$ lieu des quatre schèmes d'accord attendus, on n'a que deux schèmes. Sur quoi reposent ces deux schèmes? Pour répondre, on peut observer deux noms de même suffixe, toy « feuille de papier » et teye « chèvre ». Ces deux noms ont pour suffixe de genre $/ \mathrm{g} /$.

\begin{tabular}{|c|c|c|c|}
\hline & Dt1 & Dt2 & Pronom \\
\hline tכ8כ & : biiko & $r \varepsilon$ & ho \\
\hline teye & : biiro & $\mathrm{n}$ & hẽ \\
\hline
\end{tabular}

Bien que de suffixe $/ \mathrm{g} /$, teye adopte le schème du suffixe $/ 1 /$. On notera que tous les noms qui adoptent le schème d'accord /1/ renvoient à des êtres animés. En revanche, tous ceux qui se regroupent autour du schème d'accord de $/ \mathbf{k} /$ renvoient à des êtres non-animés.

Par conséquent, nous pouvons considérer que le koulango a deux genres, l'animé et le nonanimé. L'apparition des genres dans une langue ne se fait pas en un jour. C'est un processus long qui passe par la sélection d'une catégorie d'êtres, les êtres non 
sélectionnés étant mis ensemble dans une catégorie neutre. Quand la langue n'a que deux genres, forcément il y en un qui marque ce type de sélection, et il est caractérisé par une propriété sémantique spécifique. Ici, ce sont les êtres animés qui ont été classés dans un genre animé. Par conséquent, les êtres qui apparaissent comme n'ayant pas cette propriété sont classés comme non-animés. De fait, ils constituent le genre neutre en tant que regroupant toutes les propriétés non sélectionnées.

Avant de passer à deux genres, le koulango devait avoir quatre genres ainsi que le montrent les quatre affixes de genre. Il reste à expliquer d'une part la réduction de quatre à deux et, d'autre part, la sélection de la propriété animé.

\section{Raisons sociologiques de la réduction}

L'évolution est un principe inscrit dans toute langue et, particulièrement dans le système des genres quand il s'agit d'une langue à genres. Les raisons sont multiples; les plus connues sont l'évolution de la culture de la communauté locutrice ou celle du contact des langues.

La société koulango actuelle est une société composite ; elle résulte d'un peuplement par strates. La première strate, la couche originelle, est gur, donc elle relève d'une civilisation proche des civilisations lobi, mossi, sénoufo, etc. Dans l'aire gur, les langues sont des langues à genres, avec une moyenne de quatre genres fondées sur la subdivision principale humain/non-humain. La deuxième strate, la couche abron, appartient à une autre aire, l'aire kwa. Dans cette aire, les langues ont connu aussi un système de genres nominaux, qui a disparu dans beaucoup de langues, laissant ici et là des séquelles qui manifestent une distinction animé/non-animé (adioukrou, nzıma, entre autres).

Les Abron vivaient loin des Koulango, à l'est, dans la partie centre-est de l'actuel Ghana. Ils faisaient partie de la noblesse du royaume Gonja qui était installé avant le $19^{\mathrm{e}}$ siècle. Ce royaume vivait essentiellement des échanges commerciaux entre le nord savanier et sahélien et le sud forestier et côtier. Mais sur son flanc sud naît entre temps le royaume Ashanti autour de l'actuelle ville de Kumasi du Ghana. Suite à des conflits commerciaux, les Ashanti attaquèrent et défirent en 1860 le royaume Gonja, et dispersèrent ses occupants. Les Abron émigrèrent alors vers le nord-ouest où ils s'installèrent sur le territoire de différents peuples gur, dont celui des Koulango.

Quand cohabitation se produit entre un peuple de cultivateurs et un peuple guerrier et noble, la culture de la noblesse est dominante, les nobles voulant généralement maintenir leur culture, et les cultivateurs étant désireux de l'acquérir. Le système politique de type royal s'instaure donc en pays koulango ; les Koulango abandonnent leurs noms pour les noms abron. Mais, pour des raisons numériques, les Abron sont contraints de parler le koulango. Ils le parlent alors à leur façon et c'est cette façon qui est devenue la norme pour la première strate. Donc la langue en étude est le koulango en situation de contact avec l'abron.

49 L'abron, comme la plupart des langues kwa, a perdu ses genres. Il n'est pas prouvé qu'elle garde des séquelles de ce système sous la forme de traces d'opposition animé/non-animé, mais elle est voisine d'une langue qui en conserve, le nzıma. Il est donc possible que le locuteur abron n'ait pas éliminé le système de quatre genres du koulango, mais qu'il l'ait réduit à deux. En plus, ce locuteur est extérieur au contexte culturel gur qui oppose l'humain au non-humain et relève du contexte kwa qui, de son côté, oppose l'animé au 
non-animé. On retrouve le même processus avec les mêmes Abron accueillis par les Nafana voisins des Koulango, un peuple gur, sénoufo plus particulièrement. La langue nafana devrait avoir au moins quatre genres comme sa sœur de l'ouest, le nafara de Sinémentiali. Mais quand elle a été pris en charge par la strate abron, son système a été réduit à deux genres, l'animé et le non-animé (Yéo, 2013).

Le passage d'un système de quatre genres à un système à deux genres et la requalification des nouveaux genres par le critère de l'animation relève de la stratification historique de la société koulango.

\section{Conclusion}

51 Comme tout système, le système de genres nominaux, s'il existe, est appelé à s'adapter continuellement. Le koulango fait partie des langues dont le système des genres a évolué, passant de quatre à deux. Les quatre suffixes qui suggèrent la présence de quatre genres ont été mis en évidence. La confirmation de ces genres a suivi par l'observation des schèmes d'accord, chaque suffixe devant enclencher un schème d'accord dans le discours. Il se trouve que seuls deux schèmes d'accord sont attestés : un premier schème réalisé autour de l'ancien genre « humain » et qui regroupe désormais les noms de tous les êtres pourvus de la propriété " animé », quel que soit leur suffixe, et un second schème réalisé autour de l'ancien genre «neutre», qui regroupe les noms des êtres non pourvus de la propriété « animé ».

La réduction des genres et le basculement de l'ancienne propriété de base, humain/nonhumain, à la nouvelle propriété, animé/non-animé, résultent de la prise en charge du koulango par des locuteurs nouveaux, dont la langue première est sans genres, et qui ont contribué à restructurer la catégorie.

\section{BIBLIOGRAPHIE}

Carlson, R., 1994 : A Grammar of Supyire, Mouton de Gruyter, Berlin, New York

Corbett, G., 1991 : Gender, Cambrigde University Press, New York

Dakubu Kropp, M.E., 2010 : Noun classes : an overview of recent work in Kwa and Gur, Studies in the Languages of the Volta Basin, 6, Part 2 : Nominals, the Lexicon and Phonology, Editors : M. E. Kropp Dakubu, Nana Aba Amfo, E. K. Osam, K. Saah and Akanlig-Pare, Linguistics Departement, University of Ghana, Legon, 1-11

Dakubu Kropp, M.E., S. A. Atintono, E. A. Nsoh, 2007 : GurEnE-English Dictionary, Linguistics Departement, University of Ghana, Legon.

Delplanque, A., 1995 : Que signifient les classes nominales ? L'exemple du mooré, langue gur, Linguistique africaine, $\mathrm{n}^{\circ} 15,5-56$

Elders, S., 2008 : Grammaire kulango (parler de Bouna, Côte d'Ivoire), Rüdiger Köppe Verlag, Köln. 
Herault, G., 1982 : L'adioukrou, dans Atlas des langues kwa de Côte d'Ivoire, Tome 1, sous la Direction de Herault, ILA-ACCT, 129-153

Kaboré, R., 1985 : Essai d'analyse de la langue mùvre(Parler de Waogdgo), Département de recherche linguistique, Université Paris 7

Kra, K. A. E., 2000 : Esquisse verbal du koulango, parler de Tanda, Mémoire de Maîtrise, Université de Cocody, Abidjan.

Kra, K. A. E., 2006 : Etude phonologique et énonciative du koulango, parler de Tanda, Thèse pour le Doctorat unique, Université de Cocody, Abidjan.

Miehe, G. et W. Kerstin, éd., 2007 : Noun Class Systems in Gur Languages, vol I : Southwestern Gur Languges (without Gurunsi), Rüdiger Köppe Verlag, Köln

Roncador, M. von et G. Miehe, 1998 : Les langues gur (voltaïques). Bibliographie commentée et inventaire des appellations des langues, Rüdiger Köppe Verlag, Köln

Tchagbalé, Z., 1987 : Classes et genres en Foodo, langue Guang du Bénin, Cahiers ivoiriens de recherché linguistique, Institut de Linguistique Appliquée, Université nationale de Côte d'Ivoire, $\mathrm{n}$ $\circ 22,61-126$

Tchagbalé, Z., 2007 : Le sort des classes nominales des langues Gur, Studies in the Languages of the Volta Basin, 4, Part 1 : Nominal Constructions, Editors : M. E. Kropp Dakubu \&alii, Akanlig-Pare, E. K. Osam and K. Saah, Linguistics Departement, University of Ghana, Legon, 1-27

Tchagbalé, Z., 2010 : La problématique de la cohabitation du nombre et du genre dans les langues à genres Niger-Congo : illustration par le tem, une langue du Bassin de la Volta, Studies in the Languages of the Volta Basin, 6, Part 2 : Nominals, the Lexicon and Phonology, Editors : M. E. Kropp Dakubu et alii, Linguistics Departement, University of Ghana, Legon, 13-24

Tchagbalé, Z., 2013 : La forme de base des suffixes de genre et de nombre en sénoufo, CIRL n - 33-34, 59-75

Tchagbalé, Z., 2014 : La forme de base des affixes de genre et d'aspect dans les langues gur, CIRL n -36-A, 67-90

Yéo, K. O., 2013 : Le système des genres nafanan, un cas de changement linguistique, CIRL $\mathrm{n}$

- 33-34, 77-94

\section{RÉSUMÉS}

L'article présente un cas de langue à deux genres parmi les langues Gur. Ses auteurs postulent que tout regroupement de noms autour d'affixes différenciés est motivé sémantiquement. L'affixe autour duquel se regroupent des noms est censé exprimer une valeur sémantique, celle que les noms regroupés ont en commun. Dans les langues Niger-Congo à genres en général, en koulango en particulier, quand le nom se met au pluriel, le marqueur de pluriel se substitue au marqueur de genre, sous une forme spécifique qui permet de reconnaître le genre à travers elle. Le nombre de genres est donc déterminé par le nombre des marqueurs de genre. Le koulango dispose de quatre marqueurs de genre. Mais seuls deux schèmes d'accord sont attestés : un schème d'accord pour les noms des êtres animés, un autre pour ceux des non- animés. Les auteurs de l'article veulent montrer que le système des genres de cette langue a évolué vers sa réduction. Ils tentent ensuite d'en expliquer les raisons. 
The paper deals with two nominal genders attested in a variant of the Gur language cluster. The authors hypothesize that all noun groupings based on differentiated affixes are semantically motivated. The affix on which noun grouping obtains is supposed to convey a semantic value that acts as a common denominator for the nouns grouped together. Generally, in nominal-gender marking languages of the Niger-Congo family, and specifically the Kulango language, when the noun is plural, the plural marking affix replaces the gender marker with a specific morphology indicating clearly the nominal gender. Therefore, the number of the nominal genders is determined by the number of the gender marking morphemes. The Kulango language has four nominal gender markers. But only two agreement patterns are attested: an agreement pattern for nouns of animates and another one for non-animates. The authors of the paper want to show that the system of nominal genders in this language has evolved towards its reduction. Subsequently, they try to account for this decrease in the number nominal genders.

\section{INDEX}

Mots-clés : nom, genre, nombre, gur, affixe, suffixe

Keywords : noun, gender, number, Gur, affix, suffix

\section{AUTEUR}

\section{ZAKARI TCHAGBALÉ ET KOUAKOU APPOH ENOC KRA}

Université Félix Houphouët-Boigny, Côte d'Ivoire 